\title{
Trisomy 3, a possible recurrent cytogenetic abnormality in pediatric polymorphous post-transplant lymphoproliferative disorder (PTLD)
}

\author{
Anna Shestakova ${ }^{1}$, Narina Grove ${ }^{2}$, Jonathan Said $^{3}$, Sophie Song ${ }^{4}$, and Fabiola \\ Quintero-Rivera ${ }^{5}$ \\ ${ }^{1}$ University of California Irvine \\ ${ }^{2}$ Advanced Dermatology of Colorado \\ ${ }^{3}$ University of California, Los Angeles \\ ${ }^{4} \mathrm{UCLA}$ \\ ${ }^{5}$ David Geffen School of Medicine at UCLA
}

May 12, 2020

\begin{abstract}
Abstract: Trisomy 3 has been previously reported in association with T-cell lymphomas and less commonly in different types of non-Hodgkin B-cell lymphomas. Trisomy 3 has also been reported in two cases of pediatric post-transplant lymphoproliferative disorder (PTLD). We present comprehensive clinicopathologic review of two pediatric patients with cardiac and liver/intestinal allografts that developed polymorphous PTLD characterized by trisomy 3. Both patients had EBV viremia and EBV was positive in tissue by EBER in situ hybridization. Using karyotype analysis and fluorescence in situ hybridization, we identified trisomy 3 in both patients. Both patients responded to treatment and are now free of the PTLD. Trisomy 3 , an uncommon cytogenetic finding in PTLD, may be a recurrent cytogenetic if confirmed in a larger study of pediatric PTLD's. Further clinical follow up might help stratify significance of trisomy 3 as a prognostic factor.
\end{abstract}

Abstract: Trisomy 3 has been previously reported in association with T-cell lymphomas and less commonly in different types of non-Hodgkin B-cell lymphomas. Trisomy 3 has also been reported in two cases of pediatric post-transplant lymphoproliferative disorder (PTLD). We present comprehensive clinicopathologic review of two pediatric patients with cardiac and liver/intestinal allografts that developed polymorphous PTLD characterized by trisomy 3. Both patients had EBV viremia and EBV was positive in tissue by EBER in situ hybridization. Using karyotype analysis and fluorescence in situ hybridization, we identified trisomy 3 in both patients. Both patients responded to treatment and are now free of the PTLD. Trisomy 3 , an uncommon cytogenetic finding in PTLD, may be a recurrent cytogenetic if confirmed in a larger study of pediatric PTLD's. Further clinical follow up might help stratify significance of trisomy 3 as a prognostic factor.

\section{Introduction}

Post-transplant lymphoproliferative disorders (PTLDs) are comprised of lymphoid or plasmacytic proliferations that develop because of immunosuppression following solid organ, bone marrow, or stem cell allotransplant ${ }^{1} 2$. Vast majority of PTLDs are associated with the Epstein-Barr virus (EBV) infection and constitute EBV-driven polyclonal or monoclonal B-cell or T-cell proliferations ${ }^{3}$. There are four major subtypes of PTLDs (according to WHO 2016) - non-destructive PTLDs, monomorphic, polymorphic and classic Hodgkin lymphoma. It is important to diagnose cases as PTLD and indicate what type, because of diagnostic and prognostic implications ${ }^{3}$. Adult patients with hepatic and cardiac allografts constitute intermediate risk to develop PTLDs (approximately 1-5\%), whereas those receiving intestinal allografts have 
the highest risk (approximately 5\%) ${ }^{4}$. In pediatric patients incidence is much higher with most cases being EBV-positive polymorphous PTLD that develop during the first year following allograft transplantation ${ }^{51}$. PTLDs may involve any site of the body, with lymph nodes, lungs and gastrointestinal tract being the most common sites ${ }^{1}$. Bone marrow is involved by polymorphous PTLD in about 20 percent of cases ${ }^{6}$. Polymorphous PTLDs are composed of a heterogeneous population of lymphocytes, plasma cells and immunoblasts that efface architecture of the lymph node or extranodal tissue. Most cases of polymorphous PTLD contain numerous cells positive for EBV-encoded small RNA (EBER) ${ }^{7}$. A microarray study series of thirty five PTLDs reported overall incidence of clonal cytogenetic abnormalities in approximately $50 \%$ of cases to include gains of included 8q24, 3q27, 2p24, 5p, 9q, 11, 12q, 14q, 17q, 18q and nonrandom losses of 17p, 1p, $4 \mathrm{q}$ and $\mathrm{Xp}^{8}$. Targeted next generation sequencing studies of 50 B-cell PTLDs revealed high incidence of mutations in Lysine Methyltransferase 2D (KMT2D) and Tumor Protein p53 (TP53) genes ${ }^{9} .{ }^{1}$

Trisomy 3 has been observed in different types of lymphomas, primarily in $\mathrm{T}$ cell lymphomas, and less commonly in B cell Non-Hodgkin lymphomas, including extranodal marginal zone lymphoma of mucosaassociated lymphoid tissue (MALT) and mantle cell lymphoma ${ }^{10}$. Recently, trisomy 3 has been reported in the case of an acute megakaryoblastic leukemia ${ }^{11}$. To date there has been one publication describing two cases of pediatric post-transplant lymphoproliferative disorder with trisomy 3 as a primary chromosomal abnormality ${ }^{12}$. We present clinicopathologic overview of two cases of polymorphous PTLD and with trisomy 3 in pediatric patients with intestine/liver and cardiac allografts.

2. Materials and Methods

Immunophenotyping, in situ hybridization and clonality studies. Tissue specimens were processed for histological studies using standard methods. Immunophenotyping was performed using a panel of antibodies including CD3, CD20, PAX-5, CD138, Kappa chain, Lambda chain, CD20. EBV was demonstrated using EBER in-situ hybridization on formalin fixed paraffin-embedded tissues. For the B-cell clonality studies DNA was isolated and subjected to polymerase chain reaction using primers specific to JH immunoglobulin heavy chain, followed by gel electrophoresis and fluorescent detection. For T-cell clonality studies DNA was isolated by routine laboratory procedures and subjected to the polymerase chain reaction using primers specific for the T-cell receptor gamma locus (TRG), followed by capillary electrophoresis and fluorescent scanner detection. This technique appears to be an accurate means of detecting clonal populations of T-lymphocytes, provided the clone comprises $5 \%$ or more of the nucleated cells present in the submitted specimen.

Cytogenetic studies. The lymph node tissues were minced and squeezed to obtain a single-cell suspension. The cells were cultured without stimulation for 24 hours in Roswell Park Memorial Institute (RPMI) 1640 medium supplemented with $15 \%$ fetal calf serum. Chromosome preparation and G-banding analysis were performed following standard procedures. Karyotype and FISH results were described according to the International System for Human Cytogenetic Nomenclature (ISCN 2016) ${ }^{13}$.

Fluorescence in situ hybridization (FISH). FISH was performed on the chromosome preparations using directly labeled fluorescence painting probes for the whole chromosome 3 in patient 1 , and for the whole chromosomes 3 and $\mathrm{X}$ in patient 2. The FISH procedure was performed according to the Vysis protocol. A total of 20 metaphases were examined in each patient.

3. Results: Clinical Data and Histological, Immunophenotypic, Molecular and Cytogenetic studies

Case 1. Patient is a 16-year-old female with a history of congenital jejunoileal atresia and intestinal failureassociated liver disease who required combined orthotopic liver transplantation and intestinal transplantation at the age of 1-year-old. At the age of two patient developed refractory immune-mediated thrombocytopenic purpura, which eventually responded to splenectomy. She had a complex post-transplant course including persistent EBV viremia and a polymorphous PTLD at the age of 3-years-old, when patient presented with diffuse lymphadenopathy. At that time excisional biopsy of the supraclavicular lymph node revealed a total effacement of the nodal architecture by a polymorphous lymphoid infiltrate composed of small and large lymphoid cells, some resembling immunoblasts, as well as numerous plasma cells. Occasional atypical large 
cells were noted. No necrosis was identified. EBV was positive by EBER in situ hybridization in numerous lymphoid cells including the large cell component. The findings were consistent with the EBV-positive polymorphic post-transplant lymphoproliferative disorder (PTLD) (Figure 1). 823
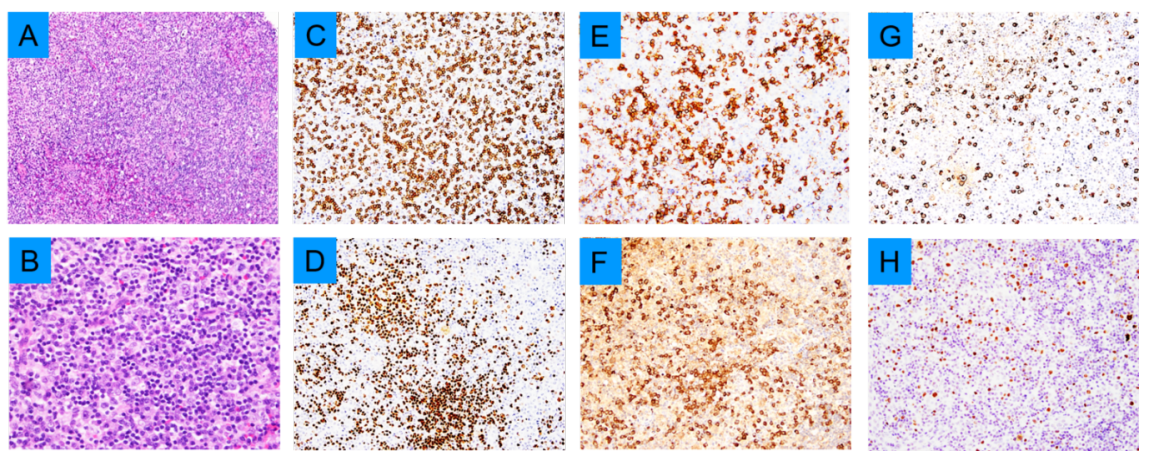

Figure 1. Patient 1: Excisional biopsy of the lymph node involved by polymorphous Post-transplant lymphoproliferative disorder. A - B. Histomorphologic findings of the lymph node biopsy reveal effacement of the nodal architecture (A. 40x) by a mixed proliferation of lymphocytes, immunoblasts, and plasma cells (B. 400x). C - F. Immunohistochemical studies highlight mixed T-cells (C. CD3), B-cells (D. PAX5), and polytypic plasma cells (E. CD138, F. Kappa, G Lambda). EBV EBER in-situ hybridization (H) highlights scattered EBV-positive cells.

Clonal B-cell rearrangement was demonstrated by the presence of a discrete peak following PCR with primers specific for the JH immunoglobulin heavy chain locus. In addition, PCR revealed prominent polyclonal background.

Concurrent bone marrow biopsy demonstrated normocellular marrow for age with infiltration of abnormal lambda light chain-restricted plasma cells ( $6 \%$ of all cells), small B-lymphocytes (18\% of all cells), and T-cells (12\% of all cells) consistent with marrow involvement by the PTLD, involving about $30 \%$ of all marrow (Figure 2 ). Histologic findings in the bone marrow resemble the lymph node. Cytogenetic studies, performed on the bone marrow aspirate, showed an abnormal female chromosome analysis comprised of two clones. 108

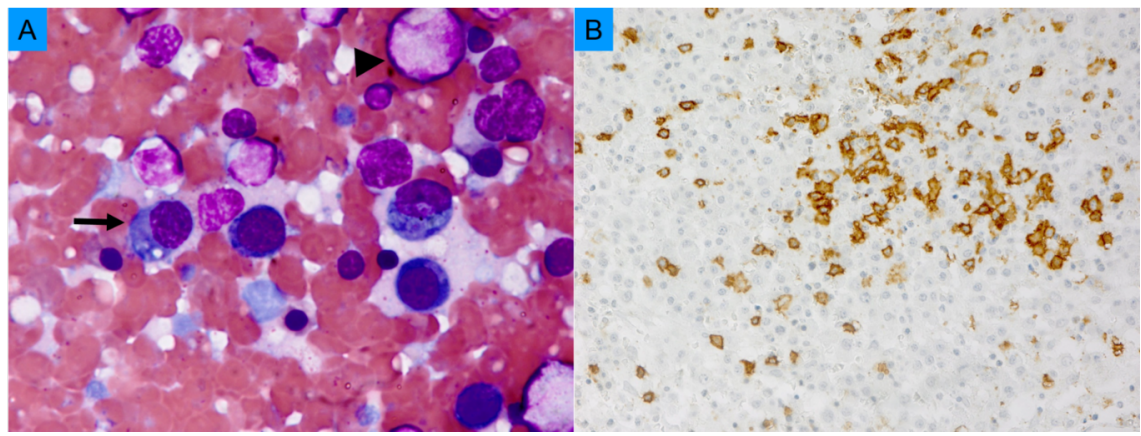

Figure 2 . Patient 1: Bone marrow showing involvement by PTLD. A. Bone marrow aspirate shows increased number of plasma cells (arrow) and large B-cells (arrowhead) (A). Immunohistochemical studies highlight both small and large B-cells (2B, CD20). 
The abnormal clone ( 8 of 20 cells) exhibited loss of one $\mathrm{X}$, and gain of an extra chromosome 3 and a marker chromosome $-47, \mathrm{X},-\mathrm{X},+3,+\operatorname{mar}[8] / 46, \mathrm{XX}[12]$. Monosomy $\mathrm{X}$ has been reported in the association with T-cell lymphoma ${ }^{14}$.

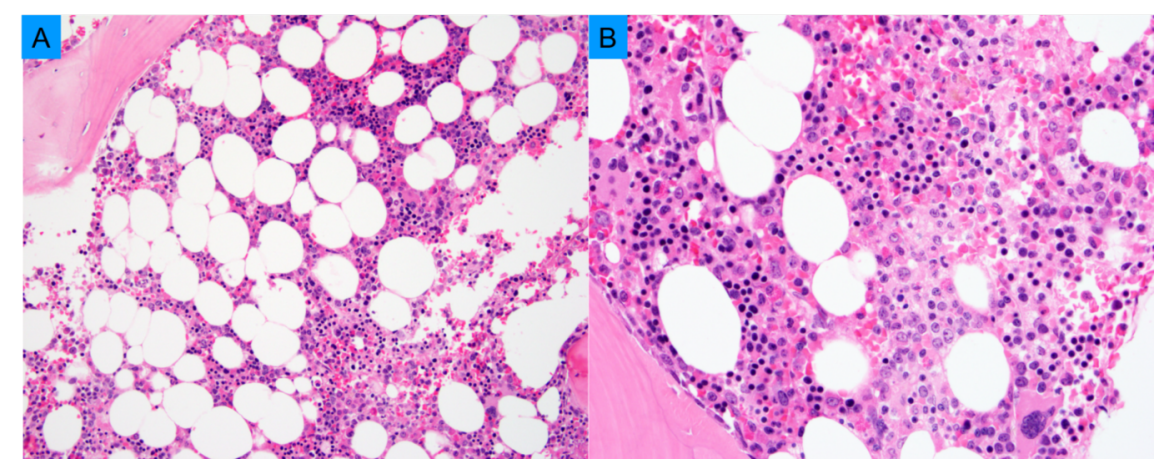

At the age of four, patient was doing well and screening bone marrow biopsy showed mildly hypocellular marrow without PTLD (Figure 3 ). Despite the persistent EBV viremia by serum PCR, EBV was negative by EBER in situ hybridization. Molecular testing did not identify a clonal population. However, cytogenetics revealed one cell with an abnormal female karyotype with trisomy 3 , similar to that previously observed in this patient 47,XX, $+3[1] / 46, \mathrm{XX}[19]$. Although one abnormal cell is considered non-clonal, due to the similar trisomy 3 finding in the previous bone marrow this finding was communicated to the clinical team to alert them for the possibility of the residual PTLD.

Figure 3. Patient 1: Bone marrow is negative for involvement by PTLD. Bone marrow biopsy shows mildly hypocellular marrow for age multilineage hematopoiesis (A. 100X and B. 400X). There is no morphologic support for involvement by PTLD.

At the age of 11 and 14 years imaging studies identified multiple new mesenteric FDG-avid mesenteric lymph nodes; however, tissue biopsy did not confirm recurrence of PTLD. Screening biopsies of the intestine did not demonstrate recurrence of the PTLD. Over the course of her disease, patient's EBV viremia was addressed by the immunosuppression reduction, Ganciclovir, and Rituximab. Currently patient is doing well and does not show signs of acute rejection or recurrent PTLD.

Case 2. The patient is a 9-year-old female born full term with a Hemoglobin (Hgb)C trait. At five weeks of age she started having cyanotic episodes and was diagnosed with left ventricular non-compaction dilated cardiomyopathy. Patient required an orthotopic heart transplant at the three months of age. Immunosuppression was mediated by the mycophenolate mofetil and tacrolimus (CellCept). Shortly after the cardiac allograft patient developed cytomegalovirus (CMV) infection. CMV viremia was successfully treated with Ganciclovir, with subsequent negative serology. At the eight months her mother noticed that she developed difficulty crawling and holding her head. At that time hydrocephalus was diagnosed and a ventriculoperitoneal shunt placed. Over the course of her clinical course patient was noted to have a marked neutropenia and reticulocytopenia refractory to the granulocyte colony stimulating growth factor Neupogen. By age four, patient required transfusion.

At age five, patient developed cervical lymphadenopathy. Although EBV viremia was noted, lymph node biopsy did not reveal PTLD. At age six, patient developed diffuse lymphadenopathy with multiple FDG-avid lymph nodes. At that time, an inguinal lymph node biopsy revealed near-total nodal effacement by a diffuse proliferation of mixed atypical lymphocytes, large transformed lymphocytes or immunoblasts, and numerous plasma cells, consistent with the involvement by polymorphous PTLD. Immunostains highlighted mixed lymphoplasmacytic cells with increased numbers of immunoblasts and polytypic plasma cells. Proliferation index was at $40 \%$, and EBV-EBER was positive by in-situ hybridization. Concurrent flow cytometry did not detect any monotypic B-cells or pan T-cell aberrancy. 


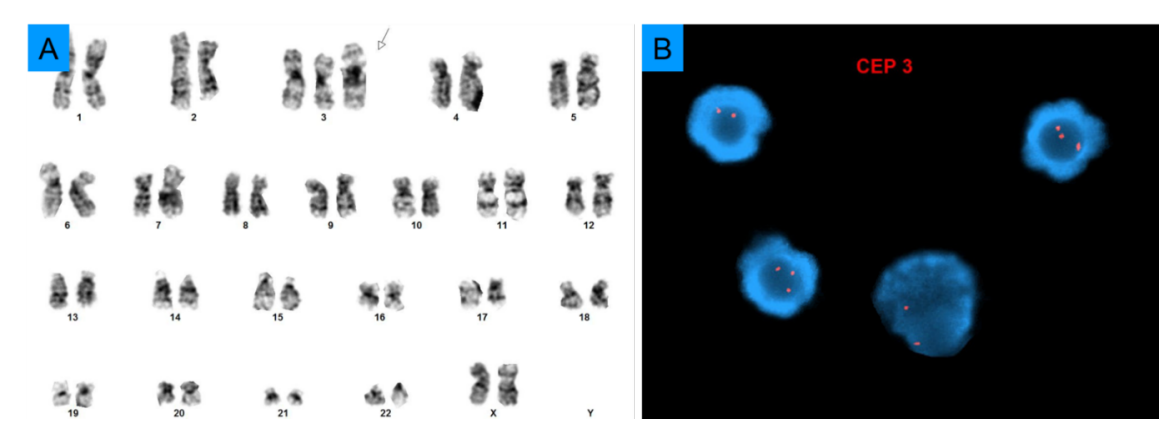

Karyotype analysis demonstrated trisomy 3 as a sole anomaly in two out of twenty analyzed cells. To confirm clonality of this finding interphase FISH was performed using a chromosome 3 centromere (CEP3) probe, which showed 25 out of 300 cells positive for trisomy 3 (Figure 4) .

Figure 4. Conventional cytogenetics (G-banded karyotype) and fluorescence in situ hybridization (FISH) studies were performed on the lymph node involved with PTLD. A. Chromosome analysis revealed $47, \mathrm{XX},+3[2] / 46, \mathrm{XX}[18]$. B. This finding was confirmed by internal FISH studies with a probe for the centromere of chromosome 3, which detected three copies of chromosome 3 in $8.3 \%(25 / 300)$ of the nuclei examined. FISH studies were performed using commercially available Vysis Centromere 3 (CEP 3 ) probe (Abbott Molecular, Des Plaines, IL), cut off value established for trisomy 3 is 1 percent.

Although bone marrow did not show histologic or immunophenotypic evidence of the involvement by PTLD, molecular studies detected clonal and oligoclonal T-cell gene rearrangements. Patient was treated with Rituximab. Currently patient is seven years old, doing well and is free of the recurrence of PTLD.

\section{Discussion}

PTLDs represent a heterogeneous, commonly EBV-driven clonal proliferation of B-cells with admixed Tcell and plasma cells ${ }^{3}$. Although PTLDs are usually diagnosed based on clinical history, morphology, and immunophenotype, clonality studies are used to aid in the diagnosis. Cytogenetics is a powerful technique to determine clonality and, if possible, interpret clinical implications of the particular clone or clones. Although molecular diagnostics dominate the era of the precision medicine, conventional chromosome analysis and FISH studies remain valuable to diagnose gross chromosomal abnormalities in PTLDs. Cytogenetics allows identification of a single cell that bears aneuploidies or large chromosomal aberrations. Furthermore, FISH studies can be utilized to confirm the clonality of findings identified by karyotype. The most common aberrations were trisomies of chromosome 9 and/or 11 associated with EBV positivity, followed by translocations involving 8q24.21 (MYC), 3q27.3 (BCL6), and 14q32.13 (IGH), 14q32.33 (TCL1) ${ }^{15}$. The identification of cytogenetic markers that either contribute to the prediction of clinical behavior or response to the treatment are important in managing patients with PTLDs. Although specific mutations have not been reported in PTLDs, aberrant somatic hypermutation was implicated in a pathogenetic process that activates protooncogenes such as such as paired box protein (PAX-5) and avian myelocytomatosis viral oncogene homolog (C-MYC) ${ }^{16}$.

Aberrations involving chromosome 3 have been described in a range of low-grade B-cell lymphomas including marginal zone lymphoma, with the incidence reaching 85 percent in splenic marginal zone lymphoma ${ }^{17}$ 18. Trisomy 3 has been reported in other types of B-cell lymphomas such as follicular lymphoma, small cell lymphocytic lymphoma, and diffuse large cell lymphoma ${ }^{19}$. Trisomy 3 has also been described in a range of T-cell lymphomas ${ }^{20}$. Chromosome 3 contains proto-oncogenes that were reported in hematologic malignancies including transducin (beta)-like 1 X-linked receptor 1 (TBL1XR1) at 3q26.32, myeloid differentiation primary response gene (88) (MYD88) at 3p22.2, GATA binding protein 2 (GATA2) at 3q21.3, ras homolog family member A (RHOA) at 3p21.31, MDS1 and EVI1 complex locus (MECOM) at 3q26.2, T cell leukemia translocation altered (TCTA) at 3p21.31 and BCL6 transcription repressor (BCL6) at 3q27.3. It is possible that the pathogenesis of PTLDs is mediated by the trisomy 3 through the increased gene dosage 
of proto-oncogenes, however precise mechanism remains elusive. Recentin vitro studies using spontaneously proliferating EBV-infected B-cells from patients with PTLD demonstrated a higher average growth rate and expression a miR-BHRF1-3, the miRNA that was reported to have transforming abilities ${ }^{21}$.

One of the patients presented here (Case 1) also had one cell with a monosomy of chromosome $\mathrm{X}$ in addition to trisomy 3 . This finding likely represents a subclone, or a secondary finding in addition to trisomy 3 . One of the ways that the loss of chromosome X might contribute to the pathogenesis of PTLD is through the loss of the copy of tumor suppressor gene. Several X-linked tumor suppressor genes including APC membrane recruitment protein 1 (AMER1) at Xq11.2 and forkhead box P3 (FOXP3) at Xp11.23 are X-linked tumor suppressor genes involved in prostate cancer ${ }^{22}{ }^{23}$. The synchronous trisomy 3 with an extra chromosome $\mathrm{X}$ has been previously reported in the pediatric PTLD ${ }^{12}$. To our knowledge, this is the first report of the pediatric polymorphous PTLD with coexisting trisomy 3 and monosomy of chromosome $\mathrm{X}$.

In the present study, we report two pediatric cases of polymorphous EBV-driven PTLDs with trisomy 3 arising in the setting of cardiac and liver/intestinal allograft. In addition, one of the patients (Case 1) had a monosomy of chromosome X. Both of the patients are doing well, which argues against poor prognostic value of trisomy 3 in PTLDs.

Further studies are necessary to characterize cytogenetic findings in the larger number of pediatric PTLD to understand the significance of trisomy 3. In addition, it is important to understand the significance of trisomy 3 with or without monosomy of chromosome X. Eventually, better understanding of the landscape of cytogenetic abnormalities in pediatric patients with PTLD will contribute to the accurate diagnosis and clinical prognosis.

Author Contributions: Diagnosis, F.Q.R., J.S., S.S.; writing - original draft preparation, A.S, N.G..; writing - review and editing, A.S., F.Q.R.; project conception, F.Q.R.

Funding: This research received no external funding.

Acknowledgments: Authors are thankful to both patients who contributed to this case report.

Conflicts of Interest: The authors declare no conflict of interest.

F.Q.R. ORCID number 0000-0002-6709-6209

\section{References}

1. Webber SA, Naftel DC, Fricker FJ, et al. Lymphoproliferative disorders after paediatric heart transplantation: a multi-institutional study. Lancet Lond Engl . 2006;367(9506):233-239. doi:10.1016/S01406736(06)67933-6

2. Uhlin M, Wikell H, Sundin M, et al. Risk factors for Epstein-Barr virus-related post-transplant lymphoproliferative disease after allogeneic hematopoietic stem cell transplantation.Haematologica . 2014;99(2):346352. doi:10.3324/haematol.2013.087338

3. Natkunam Y, Gratzinger D, Chadburn A, et al. Immunodeficiency-associated lymphoproliferative disorders: time for reappraisal? Blood . 2018;132(18):1871-1878. doi:10.1182/blood-2018-04-842559

4. Opelz G, Döhler B. Lymphomas after solid organ transplantation: a collaborative transplant study report. Am J Transplant Off J Am Soc Transplant Am Soc Transpl Surg . 2004;4(2):222-230. doi:10.1046/j.16006143.2003.00325.x

5. Dharnidharka VR. Comprehensive review of post-organ transplant hematologic cancers. Am J Transplant Off J Am Soc Transplant Am Soc Transpl Surg . 2018;18(3):537-549. doi:10.1111/ajt.14603

6. Montanari F, O'Connor OA, Savage DG, et al. Bone marrow involvement in patients with posttransplant lymphoproliferative disorders: incidence and prognostic factors. Hum Pathol . 2010;41(8):1150-1158. doi:10.1016/j.humpath.2009.11.016 
7. Nijland ML, Kersten MJ, Pals ST, Bemelman FJ, ten Berge IJM. Epstein-Barr Virus-Positive Posttransplant Lymphoproliferative Disease After Solid Organ Transplantation: Pathogenesis, Clinical Manifestations, Diagnosis, and Management. Transplant Direct . 2015;2(1). doi:10.1097/TXD.0000000000000557

8. Poirel HA, Bernheim A, Schneider A, et al. Characteristic pattern of chromosomal imbalances in posttransplantation lymphoproliferative disorders: correlation with histopathological subcategories and EBV status. Transplantation . 2005;80(2):176-184. doi:10.1097/01.tp.0000163288.98419.0d

9. Menter T, Juskevicius D, Alikian M, et al. Mutational landscape of B-cell post-transplant lymphoproliferative disorders. Br J Haematol . 2017;178(1):48-56. doi:10.1111/bjh.14633

10. Kluin PM, Schuuring E. MOLECULAR CYTOGENETICS OF LYMPHOMA. WHERE DO WE STAND IN 2010? Histopathology . 2011;58(1):128. doi:10.1111/j.1365-2559.2010.03700.x

11. Azad VF, Mehrvar A, Mehrvar N. Trisomy 3 as an acquired cytogenetic abnormality in primary acute megakaryoblastic leukemia. J Cancer Res Ther . 2018;14(Supplement):S800-S802. doi:10.4103/09731482.175430

12. Gallego MS, Bernasconi A, Dávila MT, et al. Trisomy 3 in two paediatric post-transplant lymphomas. Br J Haematol . 2002;117(3):558-562. doi:10.1046/j.1365-2141.2002.03481.x

13. McGowan-Jordan J, Simons A, Schmid M, eds. ISCN 2016: An International System for Human Cytogenomic Nomenclature . 1 edition. S. Karger; 2016.

14. Itoyama T, Chaganti RSK, Yamada Y, et al. Cytogenetic analysis and clinical significance in adult Tcell leukemia/lymphoma: a study of 50 cases from the human T-cell leukemia virus type-1 endemic area, Nagasaki. Blood . 2001;97(11):3612-3620. doi:10.1182/blood.V97.11.3612

15. Djokic M, Le Beau MM, Swinnen LJ, et al. Post-transplant lymphoproliferative disorder subtypes correlate with different recurring chromosomal abnormalities. Genes Chromosomes Cancer . 2006;45(3):313-318. doi:10.1002/gcc.20287

16. Morscio J, Dierickx D, Tousseyn T. Molecular Pathogenesis of B-Cell Posttransplant Lymphoproliferative Disorder: What Do We Know So Far? Clin Dev Immunol . 2013;2013. doi:10.1155/2013/150835

17. Salido M, Baró C, Oscier D, et al. Cytogenetic aberrations and their prognostic value in a series of 330 splenic marginal zone B-cell lymphomas: a multicenter study of the Splenic B-Cell Lymphoma Group.Blood . 2010;116(9):1479-1488. doi:10.1182/blood-2010-02-267476

18. Baliakas P, Puiggros A, Xochelli A, et al. Additional trisomies amongst patients with chronic lymphocytic leukemia carrying trisomy 12: the accompanying chromosome makes a difference. Haematologica . 2016;101(7):e299-e302. doi:10.3324/haematol.2015.140202

19. Tagawa H, Karube K, Guo Y, et al. Trisomy 3 is a specific genomic aberration of $t(14 ; 18)$ negative follicular lymphoma. Leukemia . 2007;21(12):2549-2551. doi:10.1038/sj.leu.2404817

20. Lepretre S, Buchonnet G, Stamatoullas A, et al. Chromosome abnormalities in peripheral T-cell lymphoma. Cancer Genet Cytogenet . 2000;117(1):71-79. doi:10.1016/s0165-4608(99)00151-x

21. Delecluse S, Yu J, Bernhardt K, et al. Spontaneous lymphoblastoid cell lines from patients with EpsteinBarr virus infection show highly variable proliferation characteristics that correlate with the expression levels of viral microRNAs. PloS One . 2019;14(9):e0222847. doi:10.1371/journal.pone.0222847

22. Gudmundsson J, Sulem P, Rafnar T, et al. Common sequence variants on 2p15 and Xp11.22 confer susceptibility to prostate cancer. Nat Genet . 2008;40(3):281-283. doi:10.1038/ng.89

23. Yaspan BL, McReynolds KM, Elmore JB, Breyer JP, Bradley KM, Smith JR. A haplotype at chromosome Xq27.2 confers susceptibility to prostate cancer. Hum Genet . 2008;123(4):379-386. doi:10.1007/s00439-008$0486-8$ 


\section{Figure Legends}

Figure 1. Patient 1: Excisional biopsy of the lymph node involved by polymorphous Post-transplant lymphoproliferative disorder. A - B. Histomorphologic findings of the lymph node biopsy reveal effacement of the nodal architecture (A. 40x) by a mixed proliferation of lymphocytes, immunoblasts, and plasma cells (B. 400x). C - F. Immunohistochemical studies highlight mixed T-cells (C. CD3), B-cells (D. PAX5), and polytypic plasma cells (E. CD138, F. Kappa, G Lambda). EBV EBER in-situ hybridization (H) highlights scattered EBV-positive cells.

Figure 2 . Patient 1: Bone marrow showing involvement by PTLD. A. Bone marrow aspirate shows increased number of plasma cells (arrow) and large B-cells (arrowhead) (A). Immunohistochemical studies highlight both small and large B-cells (2B, CD20).

Figure 3 . Patient 1: Bone marrow is negative for involvement by PTLD. Bone marrow biopsy shows mildly hypocellular marrow for age multilineage hematopoiesis (A. 100X and B. 400X). There is no morphologic support for involvement by PTLD.

Figure 4. Conventional cytogenetics (G-banded karyotype) and fluorescence in situ hybridization (FISH) studies were performed on the lymph node involved with PTLD. A. Chromosome analysis revealed $47, \mathrm{XX},+3[2] / 46, \mathrm{XX}[18]$. B. This finding was confirmed by internal FISH studies with a probe for the centromere of chromosome 3, which detected three copies of chromosome 3 in $8.3 \%$ (25/300) of the nuclei examined. FISH studies were performed using commercially available Vysis Centromere 3 (CEP 3) probe (Abbott Molecular, Des Plaines, IL), cut off value established for trisomy 3 is 1 percent. 

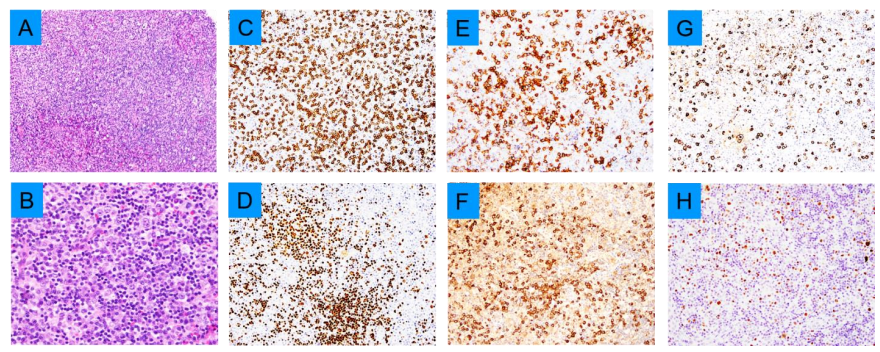

Figure 1. Patient 1: Excisional biopsy of the lymph node involved by polymorphous Post-transplant lymphoproliferative disorder. A - B. Histomorphologic findings of the lymph node biopsy reveal effacement of the nodal architecture (A. 40x) by a mixed proliferation of lymphocytes, immunoblasts, and plasma cells (B. 400x). C - F.

Immunohistochemical studies highlight mixed T-cells (C. CD3), B-cells (D. PAX5), and polytypic plasma cells (E. CD138, F. Kappa, G Lambda). EBV EBER in-situ hybridization $(\mathrm{H})$ highlights scattered EBV-positive cells. 


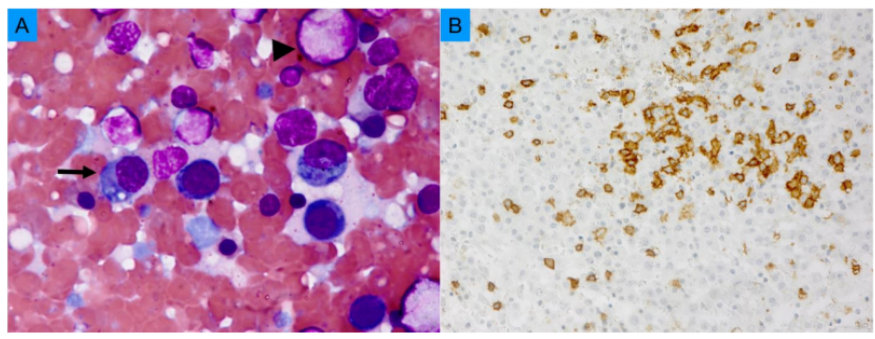

Figure 2. Patient 1: Bone marrow showing involvement by PTLD. A. Bone marrow aspirate shows increased number of plasma cells (arrow) and large B-cells (arrowhead) (A). Immunohistochemical studies highlight both small and large B-cells (2B, CD20). 


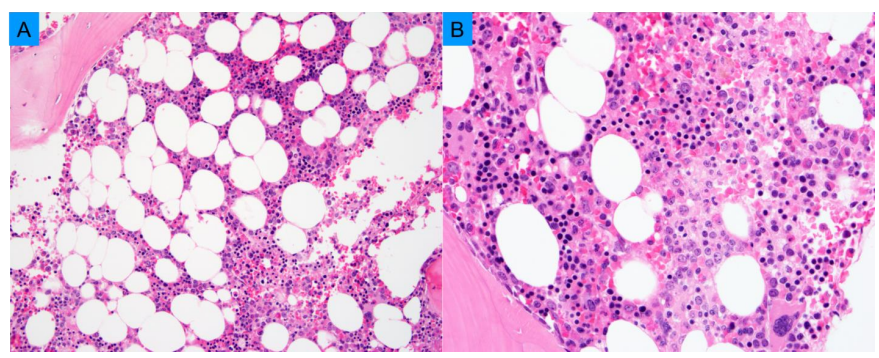

Figure 3. Patient 1: Bone marrow is negative for involvement by PTLD. Bone marrow biopsy shows mildly hypocellular marrow for age multilineage hematopoiesis (A. 100X and B. 400X). There is no morphologic support for involvement by PTLD. 


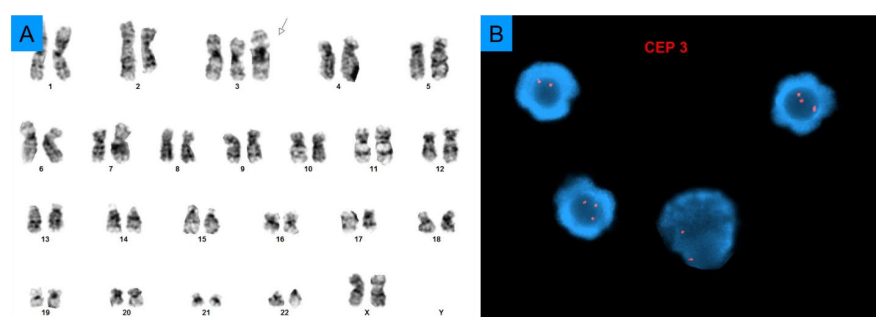

Figure 4. Conventional cytogenetics (G-banded karyotype) and fluorescence in situ hybridization (FISH) studies were performed on the lymph node involved with PTLD. A Chromosome analysis revealed $47, \mathbf{X X},+3[2] / 46, X X[18]$. B. This finding was confirmed by internal FISH studies with a probe for the centromere of chromosome 3 ,

which detected three copies of chromosome 3 in $8.3 \%(25 / 300)$ of the nuclei examined. FISH studies were performed using commercially available Vysis Centromere 3 (CEP 3 ) probe (Abbott Molecular, Des Plaines, IL), cut off value established for trisomy 3 is 1 percent. 